\title{
Decoder-Encoder LSTM for Lip Reading
}

\author{
Souheil Fenghour \\ Department of Engineering \\ London South Bank University \\ London, United Kingdom \\ fenghous@lsbu.ac.uk
}

\author{
Daqing Chen \\ Department of Engineering \\ London South Bank University \\ London, United Kingdom \\ chend@lsbu.ac.uk
}

\author{
Perry Xiao \\ Department of Engineering \\ London South Bank University \\ London, United Kingdom \\ perry.xiao@lsbu.ac.uk
}

\begin{abstract}
The success of automated lip reading has been constrained by the inability to distinguish between homopheme words, which are words have different characters and produce the same lip movements (e.g. "time" and "some"), despite being intrinsically different. One word can often have different phonemes (units of sound) producing exactly the viseme or visual equivalent of phoneme for a unit of sound. Through the use of a Long-Short Term Memory Network with word embeddings, we can distinguish between homopheme words or words that produce identical lip movements. The neural network architecture achieved a character accuracy rate of $77.1 \%$ and a word accuracy rate of $72.2 \%$.
\end{abstract}

\section{Keywords}

Deep Learning; Lip Reading; Speech Recognition; Recurrent Neural Networks; Long-Short Term Memory Networks

\section{INTRODUCTION}

Visual Speech Recognition or Lip Reading plays an important role in human communication - especially in noisy environments where audio speech recognition may be difficult. It can also be extremely useful for people whose hearing is impaired, for those who are autistic and for those suffering from language impairment not to mention that is would serve as a useful tool in assisting the police decipher CCTV footage of people speaking when audio is unavailable [4] [5] [6] [7] [8].

Automated Lip Reading remains a very challenging task and one that is made more difficult when there is no audio available for assistance. Recent Attempts have been made to automate lip reading through a variety of methodologies including Hidden Markov Models [9], Support Vector Machines [10] and Neural Networks [11] [12] [13] [14].

Automated Lip Reading has encountered many obstacles such as the insufficient supply of datasets that would be needed to train effective models, the presence of facial features and poor lighting that can inhibit feature extraction in automated lip reading systems as well as the inability to distinguish between homopheme words or words that produce identical lip movements despite being different

SAMPLE: Permission to make digital or hard copies of all or part of this work for personal or classroom use is granted without fee provided that copies are not made or distributed for profit or commercial advantage and that copies bear this notice and the full citation on the first page. To copy otherwise, or republish, to post on servers or to redistribute to lists, requires prior specific permission and/or a fee.

Conference'10, Month 1-2, 2010, City, State, Country.

Copyright 2010 ACM 1-58113-000-0/00/0010 ...\$15.00.

DOI: http://dx.doi.org/10.1145/12345.67890 and sounding different and this paper focuses on this particular problem [1] [2] [3].

The remainder of this paper is organised as follows: First in Section 2, a literature review of the main approaches to automated lip reading is given. Then in Section 4, details of the LSTM configuration are provided along with an explanation of both the dataset used to train and test the model, as well as the accuracy metrics used to evaluate accuracy of the LSTM model. In Section 5, the results of the Decoder-Encoder LSTM are discussed followed by concluding remarks given in Section 6 with suggestions for further research.

\section{RELATED WORKS}

A variety of non-deep learning based methodologies have been used to automate lip reading including Hidden Markov Models and Support Vector Machines and such methods make up the vast majority of approaches for automated lip reading. They are however far too extensive to review in this paper, but interested readers can refer to Zhou et al's [15] work for an extensive review of such methods. Deep Learning approaches to visual speech recognition have been focused on word classification. Approaches include Wand et al [14], Garg et al [13], Chung and Zisserman [12] and LipNet [11].

Wand et al. in 2016 [14] used Long Short Term Memory (LSTM) networks for lip reading, achieving an accuracy rate of $79.6 \%$ for word classification, though one major limitation to their approach was that it was speaker dependant and could not achieve as good an accuracy rate when evaluated on other speakers.

Garg et al. (2006) [13] used a pre-trained Convolutional Neural Network (CNN) based system to recognise words and phrases from the MIRACL-VC1 dataset which consists of just 10 words and phrases. An LSTM is also trained, but the CNN and LSTM need to be trained separately. The overall system attained a limited accuracy and the dataset used is relatively small and insufficient to train a model that could cover a wide enough range of subjects.

Later neural network based approaches for lip reading have deployed deep stacked networks consisting neural networks in a stacked configuration where they can be trained simultaneously. Chung and Zisserman [12] and LipNet [11] have made attempts to lip read entire sentences with both methods achieving good accuracy rates on their own respective datasets. Despite recording good accuracy both approaches are limited in their ability to classify visemes correctly as both systems are word-based classification models, and not trained specifically for the tasks of phoneme or viseme classification. In addition, both approaches are still limited in their ability to distinguish between words with identical visemes i.e. homopheme words. 
The neural network used in this paper is an LSTM based network designed to predict what word may be present given the combination of visemes that are uttered by a speaker and the prediction is performed with the use of word embeddings which allow us to predict the word spoken by context recognition. In a reallife situation, one would still need to be able to decode which visemes have been uttered by the speaker given their lip movements, but this is not the main focus of this paper.

The neural network structure is modelled according to neural machine translation [16] where stacked Recurrent Neural Networks (RNNs) are used to convert sequences of text from one language into another. Neural machine translation systems follow an encoderdecoder structure whereby the encoder reads an input sentence to encode it into a fixed length vector, and the decoder would then output a translation from the vector having been trained to maximise the probability of the "correct translation" given an input sentence. One of the main advantages of the encoder-decoder models is its ability to deal with varying lengths of input and output text sequences [17].

One significant difference between machine translation and homopheme classification is that the former tends to be one-to-one mapping whereas the latter requires one-to-many mapping because one combination of visemes can be mapped to many different words but for machine translation, context is required to decipher the identity of a spoken word through conditional probability.

\section{METHODOLOGY}

In this Section, we explain the fundamental units of speech namely, phonemes and visemes, and how they can be used to classify what is spoken upon their recognition. In addition, the dataset used to train and test our own architecture is explained, as are the algorithms used for evaluating the accuracy if the architecture.

\subsection{Phonemes and Visemes}

A phoneme, is a spoken unit of speech corresponding to a distinct sound that can be represented by an acoustic signal, whereas a viseme is the most fundamental unit of visual speech and the visual equivalent of a phoneme. According to Hazen [18], there are roughly 40 phonemes in the English language with only around a dozen distinguishable visemes. This means that several sounds can produce identical lip movements and this resulting in words that look the same when spoken, i.e. homophemes which a more common occurrence than homophone words, i.e. those that sound the same when spoken.

There are a variety of conventions which have been used to classify phonemes and visemes when analysing visual speech including Lee [19] and they all differ in their definitions of how many precise phonemes or visemes there are. In this paper, the viseme convention used is outlined in Table 1 which corresponds to Lee's [19] convention and has been shown visually in Figure 1. It appears to be the most favoured for visual speech recognition and for phonemes, the convention according to the Carnegie Mellon University Pronouncing Dictionary [20] has been used.

It was Alexander Graham Bell who first hypothesized that multiple phonemes may be visually identical on a given speaker. Because one viseme can generate multiple phonemes, the mapping of visemes to phonemes represents a one-to-many relationship [2] [21].

Words consist of phonetic symbols or phonemes which can in turn be mapped to visemes. For our neural network architecture words will be represented as combinations of visemes and every distinct combination of visemes will have its own distinct lip movements. A full visual speech recognition system would consist of an initial feature recognition stage for decoding the words that have been uttered given the representation of lip movements but this paper is focused on decoding the presence of a word or sequence of words upon the detection of distinct visemes which are based on lip movements.

Table 1. Lee and Yook's viseme convention with vowels and consonants [19]

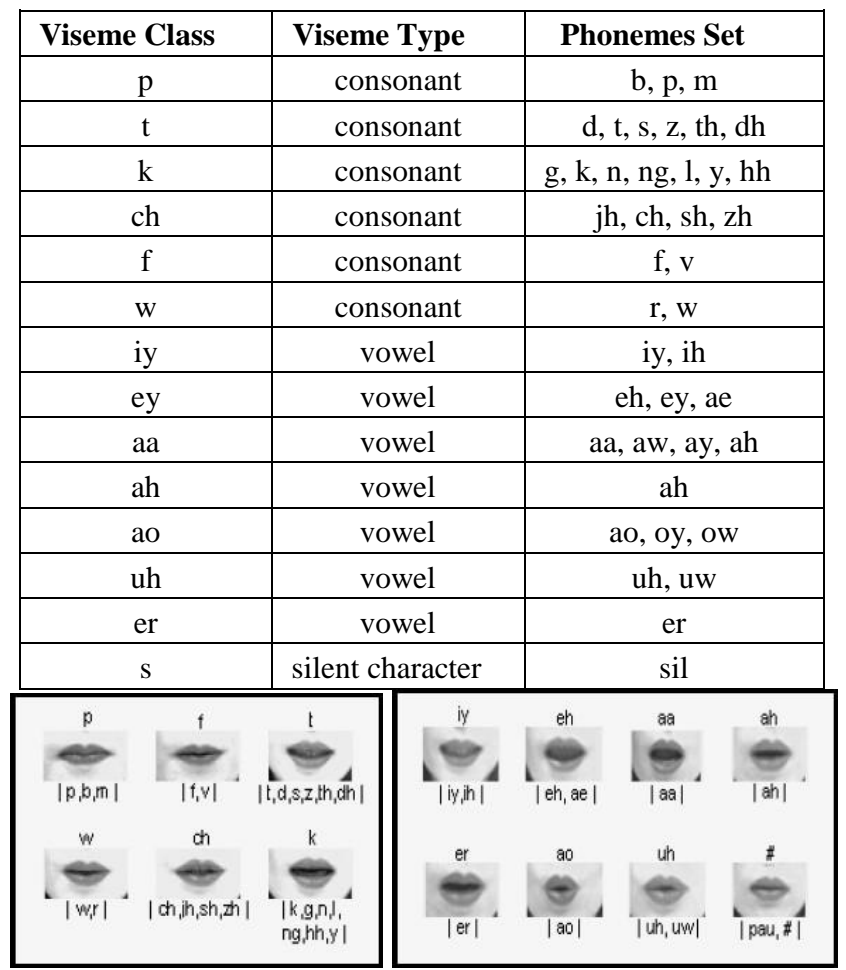

Figure 1. The six consonant visemes and the 7 vowel visemes and silent viseme. [19]

\subsection{Dataset}

The TIMIT corpus is an audio-visual dataset consisting of 630 speakers each speaking 10 different sentences giving a total of 6300 sentences available for training and testing. The speakers utter vocabulary covering the eight major dialects of American English and the overall speech has a balanced distribution of phonemes [22].

The data within the corpus consists of two parts; the first being videos that are approximately 30 seconds long that have been converted into image frames having been sampled at 25 frames per second and the second being the subtitles consisting of words that are spoken at each time step. The subtitles are word transcriptions that will have been subsampled at $16 \mathrm{kHz}$ and there will be subtitles listing each word spoken, the starting time for that word and the stopping time for that word.

For the purposes of performing homopheme detection using RNNs, the first part of the corpus will not be necessary. It is assumed that words with identical sequences of visemes share the exact same lip movements. It is the sequence of words that will be required for performing the simulations where each word will be treated like a label and its combination of visemes will be treated as a class. 
Because there are repeated sentences with one or more speakers uttering the same sentence as another, the overall dataset actually consists of 2363 distinct sentences with a vocabulary list of 6099 different words, of which there are 4764 distinct viseme combinations.

\subsection{Accuracy Metrics}

The metrics evaluating the accuracy of our architecture are character error rate(CER) and word error rate(WER).

In determining misclassifications, one has to compare the decoded speech to the actual speech and the alterations that are required to get from the decoded sentence to the actual sentence. If we look at Eq.1, $\mathrm{N}$ is the total number of words in the actual speech, $\mathrm{S}$ is the number of substitutions made for wrong classifications, I represents the number insertions made for words not picked up while $\mathrm{D}$ is the number of deletions being made for decoded words that should not be present. The word error rate WER is defined as the ratio of incorrect words decoded to the total number of words in a sample(given by Eq. 1).

Character error rate CER is calculated the same way as WER except that characters are evaluated instead of words. Furthermore, the word accuracy rate WAR and character accuracy CAR can be calculated by subtracting the either error rate from the number 1 respectively according to Eq. 2. Tables 2 and 3 give examples of how the character and word accuracies can be calculated.

$$
\begin{gathered}
W E R=\frac{(S+D+I)}{N} \\
W A R=1-W E R
\end{gathered}
$$

Table 2. Character error rates calculations for different phrases.

\begin{tabular}{|l|l|c|c|c|c|l|}
\hline Case 1 & Case 2 & S & D & I & N & CAR(\%) \\
\hline $\begin{array}{l}\text { bin blue in o six } \\
\text { now }\end{array}$ & $\begin{array}{l}\text { bin blue at l six } \\
\text { now }\end{array}$ & 3 & 0 & 0 & 3 & 85.8 \\
\hline $\begin{array}{l}\text { bin blue a x e } \\
\text { again }\end{array}$ & $\begin{array}{l}\text { bin blue at s three } \\
\text { again }\end{array}$ & 1 & 0 & 5 & 6 & 76.0 \\
\hline $\begin{array}{l}\text { lay white at e zero } \\
\text { please }\end{array}$ & $\begin{array}{l}\text { lay red in e zero } \\
\text { please }\end{array}$ & 5 & 2 & 0 & 7 & 70.8 \\
\hline
\end{tabular}

Table 3. Word error rates calculations for different phrases.

\begin{tabular}{|l|l|c|c|c|c|l|}
\hline Case 1 & Case 2 & S & D & I & N & WAR(\%) \\
\hline $\begin{array}{l}\text { bin blue in o six } \\
\text { now }\end{array}$ & $\begin{array}{l}\text { bin blue at l six } \\
\text { now }\end{array}$ & 2 & 0 & 0 & 6 & 66.7 \\
\hline $\begin{array}{l}\text { bin blue a x e } \\
\text { again }\end{array}$ & $\begin{array}{l}\text { bin blue at s three } \\
\text { again }\end{array}$ & 3 & 0 & 0 & 6 & 50.0 \\
\hline $\begin{array}{l}\text { lay white at e zero } \\
\text { please }\end{array}$ & $\begin{array}{l}\text { lay red in e zero } \\
\text { please }\end{array}$ & 2 & 0 & 0 & 6 & 66.7 \\
\hline
\end{tabular}

\subsection{Neural Network Architecture}

The neural network architecture used in this paper is shown in Figure 2. It is modelled according to neural machine translation and it is a stacked LSTM with word embeddings, a repeat vector and time-distributed network following the "encoder-decoder" model as shown in Figure 3.

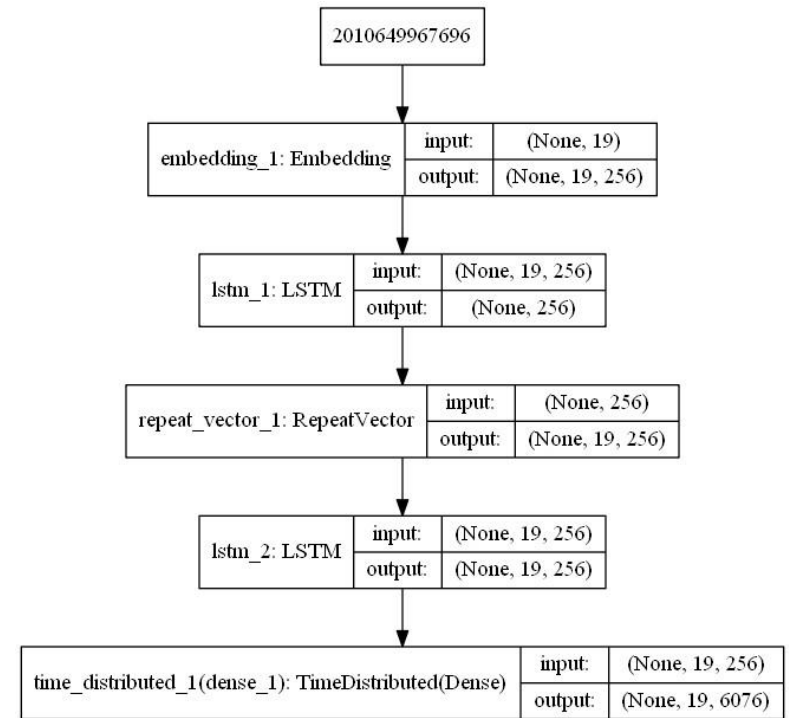

Figure 2. Structure and dimensions of the stacked LSTM configuration with the word embeddings, repeat vector and time distributed network.

Out of the 6300 sentences available to us, $90 \%$ of them will be used for training while the remainder will be used for testing. All 6300 sentences will have been converted to sequences of viseme combinations beforehand and each combination of visemes will be assigned a class label. subsequently, every sentence would be treated as a sequence of classes.

A sequence of viseme combinations forms the input of the model while the output is a sequence of words to be predicted by the network. In the same way that every viseme combination will be treated like a class, every possible word that could be predicted by the network will also be treated like a class.

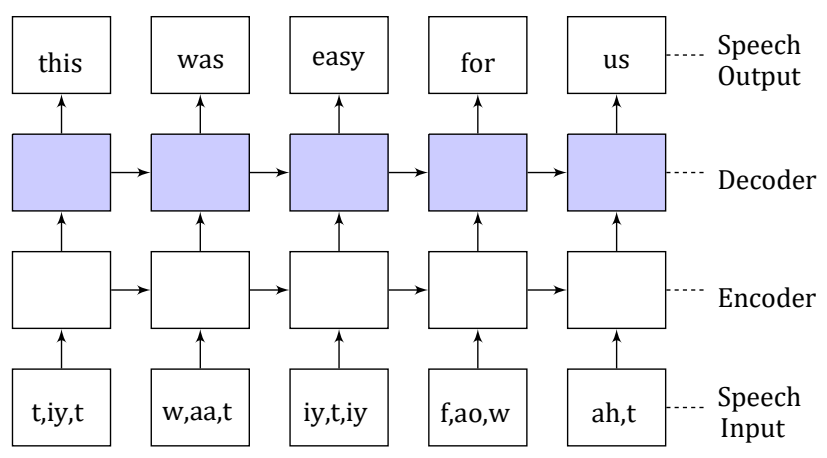

Figure 3. Stacked RNN structure with visemes as inputs and decoded words as outputs.

For an encoder-decoder framework (Eq.3 and 4), an input sentence of the form of a sequence of word vectors $\left\{x_{1}, \ldots, x_{t}\right\}$ where $x_{t}$ corresponds to a vector, is inputted into a vector $c$ with hidden state $h_{t}$ at time $t$. The vector $c$ is generated from the sequence of hidden states while $f$ and $q$ are non-linear variables.

$$
\begin{aligned}
h_{t} & =f\left(x_{t}, h_{t-1}\right) \\
c & =q\left(\left\{h_{1}, \ldots, h_{t}\right\}\right)
\end{aligned}
$$


The decoder is trained to predict next word $y_{t}$ given the context vector $c$ and all the previously predicted words $\left\{y_{1}, \ldots, y_{t}\right\}$. The decoder defines a probability $p(y)$ given in Eq. 5 over the translation $y$ by considering the joint conditional probability of all other previous words. A sentence predicted at time $t$ follows with probability $p\left(y_{t}\right)$ follows the expression given in Eq. 6 .

$$
\begin{aligned}
& p(y)=\prod_{t=1}^{T} \alpha_{i} p\left(y_{t} \mid\left\{y_{1}, \ldots, y_{t}\right\}, c\right) \\
& p\left(y_{t} \mid\left\{y_{1}, \ldots, y_{t}\right\}, c\right)=g\left(y_{t-1}, s_{t}, c\right)
\end{aligned}
$$

\section{RESULTS}

The overall stacked neural network architecture was trained on all 5670 sentences from the training set consisting of viseme combinations that were labelled by sentences composed of actual words. The network predicted the words present when combinations of visemes were inputted. Table 4 shows the overall results achieved once the network had gone through 400 epochs of iterations with sentences being grouped into batches of 60 for each iteration where an average WER and CAR of $72.2 \%$ and $77.1 \%$ respectively were achieved.

If we analyse a sample of the results in Table 5, we can see that some of the sentences follow unusual sequences where there are for example repeated words such as "that that" or "it it" and there are words decoded by the network that do not correspond to the actual words in the input sequence so there is a need to further improve the overall accuracy of the architecture.

Table 4. Results for average word-error rates and charactererror for word classifications in sentences evaluated by our architecture.

\begin{tabular}{|c|c|c|c|c|}
\hline & Epochs & Sentences & WAR(\%) & CAR(\%) \\
\hline Architecture & 400 & 630 & 72.2 & 77.1 \\
\hline
\end{tabular}

Table 5: Samples of how the sentences were decoded by the

\begin{tabular}{|c|c|c|c|}
\hline Decoded Phrase & Actual Subtitle & $\operatorname{CAR}(\%)$ & $\operatorname{WAR}(\%)$ \\
\hline $\begin{array}{l}\text { academic aptitude } \\
\text { guarantees your } \\
\text { diploma }\end{array}$ & $\begin{array}{l}\text { academic aptitude } \\
\text { guarantees your } \\
\text { diploma }\end{array}$ & 100.0 & 100.0 \\
\hline $\begin{array}{l}\text { the misprint } \\
\text { provoked } \\
\text { an immediate } \\
\text { disclaimer }\end{array}$ & $\begin{array}{l}\text { the misprint } \\
\text { provoked } \\
\text { an immediate } \\
\text { disclaimer }\end{array}$ & 100.0 & 100.0 \\
\hline $\begin{array}{l}\text { do atypical farmers } \\
\text { grow oats }\end{array}$ & $\begin{array}{l}\text { do atypical farmers } \\
\text { grow oats }\end{array}$ & 100.0 & 100.0 \\
\hline $\begin{array}{l}\text { the surplus shoes } \\
\text { were sold at a } \\
\text { discount price }\end{array}$ & $\begin{array}{l}\text { the surplus shoes } \\
\text { were sold at a } \\
\text { discount price }\end{array}$ & 100.0 & 100.0 \\
\hline $\begin{array}{l}\text { a tube a a a a the of } \\
\text { the of }\end{array}$ & $\begin{array}{l}\text { quite often } \\
\text { honeybees form a } \\
\text { majority on the } \\
\text { willow } \\
\text { catkins }\end{array}$ & 28.8 & 10.0 \\
\hline $\begin{array}{l}\text { that that it it } \\
\text { shrinking } \\
\text { shrinking faster }\end{array}$ & $\begin{array}{l}\text { but that explanation } \\
\text { is only partly } \\
\text { true }\end{array}$ & 20.0 & 14.3 \\
\hline
\end{tabular}
neural network during the testing phase.

\section{CONCLUSION}

We have addressed one of the major challenges faced in machine based lip reading which is the issue of distinguishing between homopheme words or words that produce identical lip movements. It has been demonstrated that through the use of a stacked configuration of recurrent neural networks that has been tested on a dataset designed for audio-visual speech recognition, one can detect the identify of a word in an uttered sentence provided that the viseme combination of spoken words have been accurately recognised.

Further work is required to improve the accuracy of the system and simulation results have shown that words decoded incorrectly do not share the same visemes as the true spoken words. Some decoded sentences consisted of repeated words and this something that could corrected algorithmically. The efficiency of the overall architecture is an area that could be addressed. For example, an "encoder-decoder" may not be necessary given that the number of input viseme combinations matches the number of words in each sentences meaning that we are not dealing with length variability.

\section{ACKNOWLEDGMENTS}

This project was support and funded by a joint scholarship between Chinasoft International Limited and London South Bank University.

\section{REFERENCES}

[1] A. J. Goldschen, O. N. Garcia and E. D. Petajan. (1997). Continuous automatic speech recognition by lipreading. In Motion-Based recognition.

[2] C. G. Fisher. (1968). Confusions among visually perceived consonants. Journal of Speech, Language, and Hearing Research.

[3] F. Woodward and C. G. Barber. (1960). Phoneme perception in lipreading. Journal of Speech, Language, and Hearing Research.

[4] E. T. Auer and L. E. Bernstein. (2007). Enhanced

[5] Visual Speech Perception in Individuals with Early-Onset Hearing Impairment. Journal of Speech, Language, and Hearing Research.

[6] R. Campbell and T. E. Mohammed. (2010).

[7] Speechreading for information gathering: a survey of scientific sources. Deafness Cognition and Language Research Centre.

[8] R. Bowden et al. (2013). Recent developments in automated lip-reading. Proceedings of SPIE - The International Society for Optical Engineering.

[9] M. Bohning et al. (2002). Audiovisual speech perception in Williams syndrome. Neuropsychologia.

[10] J. Leybaert et al. (2014). Atypical audio-visual speech perception and McGurk effects in children with specific language impairment. Front Psychol.

[11] C. Neti et al. (2000). Audio visual speech recognition. Technical report IDIAP.

[12] K. Saenko, K. Livescu, J. Glass, and T. Darrell. (2005). Production Domain Modeling Of Pronunciation For Visual Speech Recognition. ICASSP. 
[13] Y. M. Assael, B. Shillingford, S. Whiteson and N. de Freitas. (2016). LipNet: End-to-End sentence-Level Lipreading. ICLR Conference.

[14] J. S. Chung, A. Zisserman, A. Senior and O. Vinyals. (2016). Lip Reading Sentences in the Wild. IEEE

[15] Conference on Computer Vision and Pattern Recognition.

[16] A. Garg, J. Noyola, and S. Bagadia. (2016). Lip reading using CNN and LSTM. Technical report Stanford University - CS231n project report.

[17] M. Wand, J. Koutnik, and J. Schmidhuber. (2016). Lipreading with long short-term memory. In IEEE International Conference on Acoustics, Speech and Signal Processing pp 6115-6119.

[18] Z. Zhou, G. Zhao, X. Hong and M. PietikAd'inen. ` (2014). A review of recent advances in visual speech decoding. Image and vision computing.

[19] B. Bahdanau, K. Cho, and Y Bengio. (2014). Neural Machine Translation by Jointly Learning to Align and Translate. ArXiv. 1409.

[20] Y. Wu et al. (2016). Google's Neural Machine Translation System: Bridging the Gap between Human and Machine Translation.

[21] T. J. Hazen, K. Saenko, C. La and J. R. Glass. (2004). A segment-based audio-visual speech recognizer: data collection, development, and initial experiments. Proceedings of the 6th International Conference on Multimodal Interfaces.

[22] S. Lee and D. Yook. (2002). Audio-to-Visual

[23] Conversion Using Hidden Markov Models. In

[24] Proceedings of the 7th Pacific Rim International Conference on Artificial Intelligence: Trends in Artificial Intelligence.

[25] R. Treiman, B. Kessler and S. Bick. (2001). Context sensitivity in the spelling of English vowels. Journal of Memory and Language.

[26] F. DeLand. (1931). The story of lip-reading, its genesis and development.

[27] L. Lamel, R, H. Kassel and S. Seneff. (1989). Speech database development: Design and analysis of the acousticphonetic corpus. Proceedings of the DARPA Speech Recognition Workshop. 